\title{
A novel calibration algorithm for five-hole pressure probe
}

\author{
Akshoy Ranjan Paul ${ }^{1 *}$, Ravi Ranjan Upadhyay ${ }^{2}$, Anuj Jain ${ }^{1}$

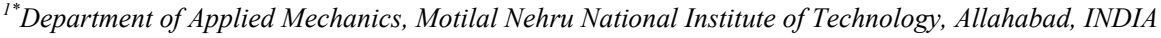 \\ ${ }^{2}$ Department of Mechanical Engineering, Motilal Nehru National Institute of Technology, Allahabad, INDIA \\ "Corresponding Author: e-mail: arpaul2k@yahoo.co.in,Tel +91-532-2271212
}

\begin{abstract}
Five-hole pneumatic pressure probes are used to carry out the steady-state measurements of three components of velocity, inflow angles, static and total pressures simultaneously for a point in a flow field. Various calibration algorithms for five-hole probes are studied in this paper as reported in the literature. Authors have defined non-dimensional pressure coefficients in different ways. Based on certain limitations, a new set of pressure coefficients are proposed in this paper, which overcome the limitations in the literature and gives less computational errors in calculating the flow parameters. In this method, the influence of pressure recorded by central hole $\left(P_{5}\right)$ is considered in defining these coefficients. From 4th order regression analysis, the average values of $r^{2}$ parameters for all zones are 0.9979 and 0.9910 for $\alpha$ and $\beta$ respectively, and which are even better than that reported in the all existing methods
\end{abstract}

Keywords: Multi-hole pressure probe, Five-hole probe, calibration, three-dimensional flow, data reduction technique.

\section{Introduction}

Five-hole probes are used to measure the three components of velocity, inflow angles, static and total pressures simultaneously for a point in a flow field. Measurement by such probe is categorized into two methods; the balancing one (null method) and the fixed one (non-null method). In the null method, the probe is rotated so that the pressures from the holes are balanced in order to measure the flow direction, static and total pressure. This method, however, requires a complex probe traversing mechanism for the rotation of the probe, which often causes difficulty to install the measurement unit into the given flow field. In the non-nulling method, the probe does not require any rotation. Many details on the use of pressure probes can be found in Bryer and Pankhurst (1971). Calibration of a five-hole probe requires subjecting the probe to a known flow field at different combinations of yaw and pitch angles. Pressures at all five holes are measured for each angular position of the probe. The measured data are represented as dimensionless pressure coefficients. A data reduction technique is then employed to correlate known flow field with the pressure coefficients. Various calibration algorithms for five-hole probes are proposed. These algorithms differ in the manner in which the pressure coefficients are defined and the data reduction technique employed.

Various calibration algorithms for five-hole probes are studied in this paper as reported by Treaster and Yocum (1979), Pisasale and Ahmed (2002), and Gallington (1980). In these papers, the authors defined non-dimensional pressure coefficients in different ways. Considering 4th order regression analysis, the goodness of fit $\left(r^{2}\right)$ parameters as per the coefficients defined in (Pisasale and Ahmed, 2002) are 0.9772 and 0.9476 for pitch $(\alpha)$ and yaw $(\beta)$ angles respectively, which are much lower, considering the fact that the same parameters reported as per the coefficients defined in [1] are 0.9957 and 0.9910 . It was observed by Zillac (1993) that the flow on the lee side of most probes begins to separate the flow angles approximately $30^{\circ}$. To avoid this situation, a sectoring scheme as described by Gallington (1980) may be adopted, which selects combinations of holes for which the flow is attached. Considering 4th order regression analysis, the average values of $r^{2}$ parameters for all zones are 0.9975 and 0.9907 for $\alpha$ and $\beta$ respectively, which exhibits the usefulness the adopting sector scheme. But it has been felt that for any combination of pitch and yaw angles, the influence of pressure sensed by the central hole of the probe $\left(P_{5}\right)$ can not be neglected as did in (Gallington, 1980). Keeping the limitations of these coefficients as discussed in view, a new set of pressure coefficients are proposed in this 
paper, which overcome the limitations and gives less computational errors in calculating the flow parameters. In this method, the influence of pressure recorded by central hole $\left(P_{5}\right)$ is considered in defining these coefficients.

\section{Pressure Normalization Techniques}

From the literature review of the calibration of five-hole probes, it is revealed that there are four ways to define non-dimensional pressure coefficients. Krause and Dudzindki (1969) first described the pressure coefficient normalization technique, for a five-hole probe with centre hole pressure labeled as $P_{5}$ and the off-axis holes labeled as $P_{1}, P_{2}, P_{3}$ and $P_{4}$, the four pressure coefficients are given by
$C_{P_{\alpha}}=\frac{P_{1}-P_{3}}{P_{5}-\bar{P}}$
$C_{P_{\beta}}=\frac{P_{2}-P_{4}}{P_{5}-\bar{P}}$
$C_{P_{\text {total }}}=\frac{P_{5}-P_{\text {total }}}{P_{5}-\bar{P}}$
$C_{P_{\text {satic }}}=\frac{\bar{P}-P_{\text {static }}}{P_{5}-\bar{P}}$

where $\bar{P}=\left(P_{1}+P_{2}+P_{3}+P_{4}\right) / 4$

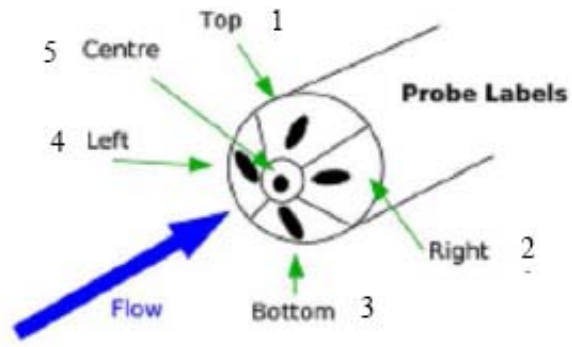

Figure 1. Nomenclature of Five-hole probe

Normalization by $P_{5}-\bar{P}$ has been incorporated in the majority of the multi-hole probe calibration procedures developed in subsequent years (Treaster and Yocum, 1979; Sitaram and Treaster, 1985; Westphal et al, 1987). The advantage of this method lies in its simplicity and provides a good foundation to understand the operation of multi-hole probes. The principal disadvantage of this method is that at large flow angles measurements become increasingly inaccurate as it encounters the problem of singularity.

A different method of extending the range of calibration is suggested by Pisasale and Ahmed (2002), which reportedly removes the problem of singularity up to flow angles of $\pm 75^{\circ}$. In this work, the denominator $P_{5}-\bar{P}$ as described in the method of Krause and Dudzindki (1969) and later restated in Treaster and Yocum (1979) was replaced by $P_{5}-P_{\text {static }}+A q$, which successfully allows calibration to much higher angles of pitch and yaw, while maintaining the simplicity of the original procedure. The results obtained using the coefficients as defined in Pisasale and Ahmed (2002), however does not exhibit much improvement for the calibration data taken in the range of $\pm 30^{\circ}$ pitch and $\pm 45^{\circ}$ yaw angles. The correct assessment of A value influences a lot in determining the calibration coefficients. Considering 4th order regression analysis, the goodness of fit $r^{2}$ parameters are 0.9772 and 0.9476 for $\alpha$ and $\beta$ respectively, which are much lower, considering the fact that the same parameters reported as per the coefficients defined in Treaster and Yocum (1979) are 0.9957 and 0.9910 .

It was observed by Zillac (1993) that the flow on the lee side of most probes begins to separate the flow angles approximately $30^{\circ}$. To avoid this situation, a sectoring scheme as described by Gallington (1980) may be adopted, which selects combinations of holes for which the flow is attached. According to this sectoring scheme, the entire calibration zone is divided into five parts, one central zone (zone-5) and four side zones (zone 1 to 4). For a particular zone, the pressure coefficients are formed by using the hole that is sensing the maximum pressure and the holes adjacent to the maximum pressure hole. This process is referred to as zonal discrimination and for a five-hole probe; the pressure coefficients in various zones as per Gallington (1980) are defined as follows.

Zone-1:

$D=P_{1}-\frac{P_{2}+P_{4}}{2}$

Zone-2:

$C_{P_{\alpha}}=\frac{P_{1}-P_{5}}{D}$
$D=P_{2}-\frac{P_{1}+P_{3}}{2}$

$C_{P_{\alpha}}=\frac{P_{1}-P_{3}}{D}$
Zone-3:

$D=P_{3}-\frac{P_{2}+P_{4}}{2}$

$C_{P_{\alpha}}=\frac{P_{5}-P_{3}}{D}$
Zone-4:

$D=P_{4}-\frac{P_{1}+P_{3}}{2}$

$C_{P_{\alpha}}=\frac{P_{1}-P_{3}}{D}$
Zone-5:

$\bar{P}=\frac{P_{1}+P_{2}+P_{3}+P_{4}}{4}$

$C_{P_{\alpha}}=\frac{P_{1}-P_{3}}{P_{5}-\bar{P}}$ 


$$
\begin{aligned}
& C_{P_{\beta}}=\frac{P_{2}-P_{4}}{D} \\
& C_{P_{\beta}}=\frac{P_{2}-P_{5}}{D} \\
& C_{P_{\beta}}=\frac{P_{2}-P_{4}}{D} \\
& C_{P_{\beta}}=\frac{P_{5}-P_{4}}{D} \\
& C_{P_{\beta}}=\frac{P_{2}-P_{4}}{P_{5}-\bar{P}} \\
& C_{P_{\text {total }}}=\frac{P_{1}-P_{\text {total }}}{D} \\
& C_{P_{\text {otola }}}=\frac{P_{2}-P_{\text {total }}}{D} \\
& C_{P_{\text {total }}}=\frac{P_{3}-P_{\text {total }}}{D} \\
& C_{P_{\text {total }}}=\frac{P_{4}-P_{\text {total }}}{D} \\
& C_{P_{\text {total }}}=\frac{P_{5}-P_{\text {total }}}{P_{5}-\bar{P}}
\end{aligned}
$$

The results achieved using the coefficients as defined in Gallington (1980) shows much improvement. Considering 4th order regression analysis, the average value of goodness of fit $r^{2}$ parameters for all zones are 0.9975 and 0.9907 for $\alpha$ and $\beta$ respectively, which exhibits the usefulness the adopting sector scheme.

But it has been felt that for any combination of pitch and yaw angles, the influence of pressure sensed by the central hole of the probe $\left(P_{5}\right)$ cannot be neglected as did in Gallington (1980). Keeping the limitations of these coefficients as discussed in view, the authors have proposed a new set of coefficients, which overcome the limitations and gives less computational errors in calculating the flow parameters. In this method, the influence of pressure recorded by central hole $\left(P_{5}\right)$ is considered in defining these coefficients. As per the new proposed method, the pressure normalization coefficients for various zones are defined below.
Zone-1:
Zone-2:
Zone-3:
Zone-4:
Zone-5:
$\bar{P}=\frac{P_{4}+P_{5}+P_{2}}{3}$
$\bar{P}=\frac{P_{1}+P_{5}+P_{3}}{3}$
$\bar{P}=\frac{P_{4}+P_{5}+P_{2}}{3}$
$\bar{P}=\frac{P_{1}+P_{5}+P_{3}}{3}$
$\bar{P}=\frac{P_{1}+P_{2}+P_{3}+P_{4}}{4}$
$D=P_{1}-\bar{P}$
$D=P_{2}-\bar{P}$
$D=P_{3}-\bar{P}$
$C_{P_{\alpha}}=\frac{P_{1}-P_{5}}{D}$
$C_{P_{\alpha}}=\frac{P_{1}-P_{3}}{D}$
$C_{P_{\alpha}}=\frac{P_{5}-P_{3}}{D}$
$D=P_{4}-\bar{P}$
$D=P_{5}-\bar{P}$
$C_{P_{\beta}}=\frac{P_{2}-P_{4}}{D}$
$C_{P_{\beta}}=\frac{P_{2}-P_{5}}{D}$
$C_{P_{\beta}}=\frac{P_{2}-P_{4}}{D}$
$C_{P_{\alpha}}=\frac{P_{1}-P_{3}}{D}$
$C_{P_{\alpha}}=\frac{P_{1}-P_{3}}{D}$
$C_{P_{\text {total }}}=\frac{P_{1}-P_{\text {total }}}{D}$
$C_{P_{\text {toal }}}=\frac{P_{2}-P_{\text {total }}}{D}$
$C_{P_{\text {total }}}=\frac{P_{3}-P_{\text {total }}}{D}$
$C_{P_{\beta}}=\frac{P_{5}-P_{4}}{D}$
$C_{P_{\beta}}=\frac{P_{2}-P_{4}}{D}$
$C_{P_{\text {staic }}}=\frac{\bar{P}-P_{\text {static }}}{D}$
$C_{P_{\text {staic }}}=\frac{\bar{P}-P_{\text {static }}}{D}$
$C_{P_{\text {static }}}=\frac{\bar{P}-P_{\text {static }}}{D}$
$C_{P_{\text {toata }}}=\frac{P_{4}-P_{\text {total }}}{D}$
$C_{P_{\text {toata }}}=\frac{P_{5}-P_{\text {total }}}{D}$
$C_{P_{\text {staic }}}=\frac{\bar{P}-P_{\text {static }}}{D}$
$C_{P_{\text {staic }}}=\frac{\bar{P}-P_{\text {static }}}{D}$

\section{Data Reduction Techniques}

Calibration of a five-hole probe requires subjecting the probe to a known flow field. Pressures at all five holes are measured for each angular position of the probe during the calibration. A calibration data reduction method is then employed, such as the polynomial curve-fit method of Gallington (1980) and Gerner et al (1984) or the direct-interpolation method of Zillac (1993). A particular data set is used for all combinations of pressure normalization techniques and data reduction techniques to find the perfect combination of the pressure normalization technique and data reduction technique. Error analysis is helpful in this regard, where the following parameters are useful in deciding the perfect combination:

Uncertainty, $\sigma_{\theta}=\sqrt{\frac{1}{n} \sum_{i=1}^{n}\left(\theta_{e, i}-\theta_{a, i}\right)^{2}} ;$ Standard error, $S E=\frac{\sigma_{\theta}}{\sqrt{n}}$

Goodness of fit or coefficient of determination, which is commonly known as $r^{2}$ and is expected to be close to 1. It compares estimated $\left(\theta_{e}\right)$ and actual $\left(\theta_{a}\right)$ values, and ranges in value from 0 to 1 . If it is 1 , there is a perfect correlation in the sample- there is no difference between the estimated and the actual value. At the other extreme, if the coefficient of determination is 0 , the regression equation is not helpful in predicting a $y$-value.

\section{Experimental Procedure}

The calibration of the five-hole probe in discussion was conducted in a low-speed open-circuit type internal flow generation facility. A $4 \mathrm{~mm}$ diameter five-hole probe was fabricated with the tip of the probe shaped as perpendicular pyramid. The probe was comprised of five close-packed $0.8 \mathrm{~mm}$ diameter stainless steel tubes fitted into a $4 \mathrm{~mm}$ diameter outer stainless steel sleeve. Among the five tubes, one forward-facing tube at the centre (denoted as 5), two chamfered side tubes in horizontal axis (denoted as 2 and 4), and remaining two side tubes in vertical axis (denoted as 1 and 3) as shown in figure 1. The tips of four side tubes are 
chamfered to $\pm 45^{\circ}$ by means of a special jig-and-fixture. The four chamfered tubes were assembled by soldering around the central tube. The length of the five-hole probe is $0.6 \mathrm{~m}$.

A probe traversing mechanism was designed and constructed, which allows the rotation of the probe $\pm 90^{\circ}$ in pitch $(\alpha)$ plane and $\pm 150^{\circ}$ in yaw $(\beta)$ plane with an angular resolution of $\pm 0.5^{\circ}$. Free stream velocity of air was monitored at $22 \mathrm{~m} / \mathrm{s}$ by a standard pitot tube connected to a microprocessor based digital micromanometer (FCO510) with a range of 0-200 mm of water column from Furness Controls Ltd., UK. The five pneumatic pressure tubing of five-hole probe were connected to a digital pressure scanning box (FCS421) from the same company, which enabled to measure multi-pressure source using just one pressure measuring instrument (digital micromanometer in this case). The fluctuating pressure signals were typically averaged over a period of 5 seconds time to allow for conditions to reach steady-state. Using the probe traversing mechanism, the pitch and yaw angles of the probe were changed by $5^{\circ}$ increments in range of $\pm 30^{\circ}$ and $\pm 45^{\circ}$ respectively.

\section{Results and Discussion}

The paper attempts to investigate a three pressure normalization techniques in combination with three data reduction techniques reported in the multi-hole pressure probe literatures. A particular set of calibration data for a five-hole probe was generated experimentally and used for analyzing the possible combinations of pressure normalization and data reduction techniques. A new pressure reduction technique is also presented in this paper, where the effect of centre-hole pressure $\left(P_{5}\right)$ is also included in defining all pressure normalization parameters.

\section{1 Sector Map}

Fig. 2 shows a sector map for five-hole probe indicating the hole, which registers maximum pressure for a particular pitch and yaw angles. It reveals that the central hole (hole-5) covers the widest range of pitch and yaw angles among all the five holes. However at larger pitch and yaw angles, the peripheral holes (hole-1 to 4) are likely to sense the total pressure, and hence pressure sensed by the peripheral holes at their corresponding location is maximum. Asymmetry in sector map depicts the non-symmetric nature of the five-hole probe due to fabrication limitation.

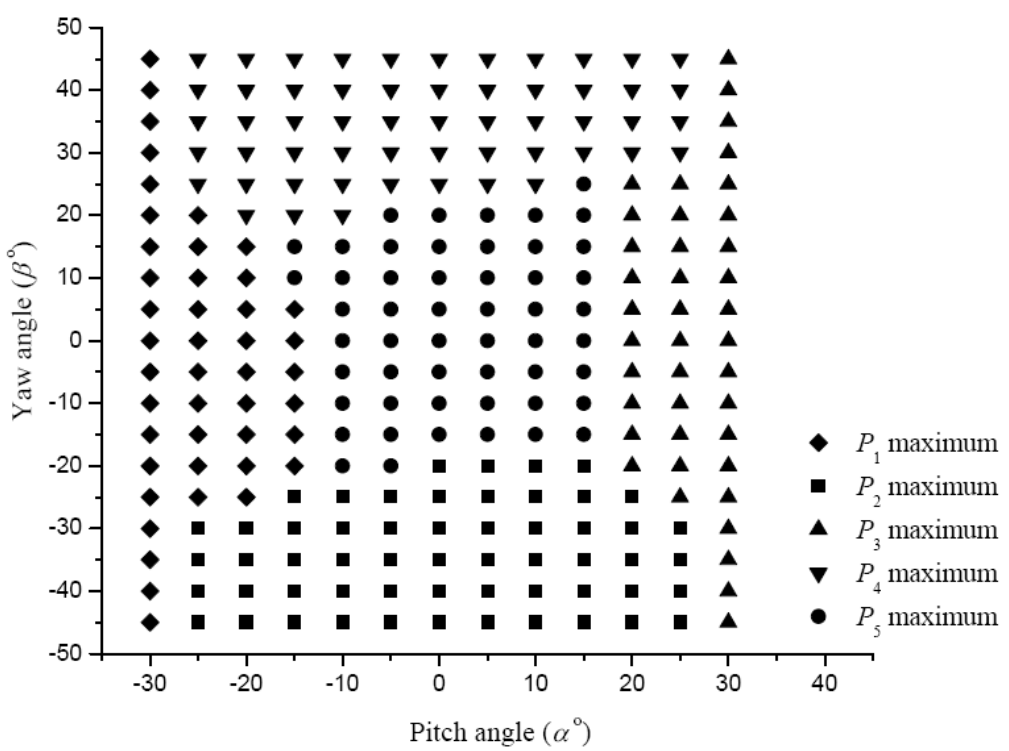

Figure 2. Sector map of five-hole probe

\subsection{Comparison of Surface Fit Methods}

'Goodness of fit' or 'coefficient of determination', which is commonly known as $r^{2}$ and is expected to be close to 1. It compares measured $\left(\theta_{m}\right)$ and computed $\left(\theta_{c}\right)$ values, and ranges in value from 0 to 1 . If it is 1 , there is a perfect correlation in the samplethere is no difference between the measured and the computed value. At the other extreme, if the coefficient of determination is 0 , the regression equation is not helpful in predicting a value.

Considering fourth order regression analysis (a variant of polynomial surface fit method), the $r^{2}$ as per the coefficients defined by Pisasale and Ahmed (2002) are 0.9772 and 0.9476 for pitch $(\alpha)$ and yaw $(\beta)$ angles respectively, which are lower, considering the fact that the same parameters reported as per the coefficients defined in Treaster and Yocum (1979) are 0.9957 and 0.9910 for the same range of $\alpha$ and $\beta$. In case of total and static pressures, the values of $r^{2}$ are reported even better as per Treaster and Yocum (1979). 
The statistical results achieved using the coefficients as defined by Gallington (1980) shows further improvement. Considering fourth order regression analysis, the average values of $r^{2}$ parameters for all zones are 0.9975 and 0.9907 for $\alpha$ and $\beta$ respectively, which exhibits the usefulness the adopting sector scheme. The statistical results furnished in table 1 also reveal that the goodness of fit $\left(r^{2}\right)$ values in the computation of total and static pressures are less as compared to that for pitch and yaw angles. Therefore focus is made on better prediction of total and static pressures.

The exact uncertainty of the velocity and pressure measurement is a function of not only the curve-fits, which are dependent upon the probe design, but upon the accuracy of the five pressure measurements. The error analysis done here is based on the pressure normalization techniques and data reduction methods chosen, and hence is considered as 'computational' errors. It has no connection with the 'experimental' accuracy of the instrument or 'experimental' errors involved during data generation. Table 1 shows the statistical summary of all combinations of pressure normalization and data reduction techniques used in the study.

Table 1: Statistical summary of all combinations of pressure normalization and data reduction techniques

\begin{tabular}{|c|c|c|c|c|c|c|}
\hline \multirow{2}{*}{$\begin{array}{l}\text { Pressure } \\
\text { normalization } \\
\text { techniques }\end{array}$} & \multirow[t]{2}{*}{$\begin{array}{l}\text { Data reduction } \\
\text { techniques }\end{array}$} & \multirow[t]{2}{*}{$\begin{array}{l}\text { Statistical } \\
\text { Parameters }\end{array}$} & Pitch & Yaw & $\begin{array}{l}\text { Total } \\
\text { Pressure } \\
\end{array}$ & $\begin{array}{l}\text { Static } \\
\text { Pressure }\end{array}$ \\
\hline & & & Degree & Degree & $\begin{array}{l}\text { mm of } \\
\mathrm{H}_{2} \mathrm{O} \\
\end{array}$ & $\begin{array}{l}\mathrm{mm} \text { of } \\
\mathrm{H}_{2} \mathrm{O}\end{array}$ \\
\hline \multirow{5}{*}{$\begin{array}{l}\text { Treaster and } \\
\text { Yocum (1979) }\end{array}$} & \multirow{3}{*}{$\begin{array}{l}\text { Polynomial Curve-fit } \\
\text { (4th order Regression) }\end{array}$} & Uncertainty & 1.2232 & 2.0699 & 0.2343 & 0.0824 \\
\hline & & Standard error & 0.0863 & 0.1460 & 0.0384 & 0.0058 \\
\hline & & $r^{2}$ value & 0.9957 & 0.9910 & 0.9417 & 0.9545 \\
\hline & \multirow{2}{*}{$\begin{array}{l}\text { Direct Interpolation } \\
\text { (Akima IMSL) }\end{array}$} & Uncertainty & 0.4409 & 0.3321 & 0.0459 & 0.0116 \\
\hline & & Standard error & 0.0321 & 0.0234 & 0.0032 & 0.0008 \\
\hline \multirow{5}{*}{$\begin{array}{l}\text { Pisasale and } \\
\text { Ahmed (1002) }\end{array}$} & \multirow{3}{*}{$\begin{array}{l}\text { Polynomial Curve-fit } \\
\text { (4th order Regression) }\end{array}$} & Uncertainty & 3.0493 & 6.2824 & 0.0706 & 0.2310 \\
\hline & & Standard error & 0.1873 & 0.3859 & 0.0043 & 0.0142 \\
\hline & & $r^{2}$ value & 0.9772 & 0.9476 & 0.8556 & 0.7968 \\
\hline & \multirow{2}{*}{$\begin{array}{l}\text { Direct Interpolation } \\
\text { (Akima IMSL) }\end{array}$} & Uncertainty & 0.3198 & 0.1899 & 0.0176 & 0.0082 \\
\hline & & Standard error & 0.0228 & 0.0151 & 0.0011 & 0.0005 \\
\hline \multirow{5}{*}{$\begin{array}{l}\text { Gallington } \\
\text { (1980) }\end{array}$} & \multirow{3}{*}{$\begin{array}{l}\text { Polynomial Curve-fit } \\
\text { (4th order Regression) }\end{array}$} & Uncertainty & 0.4026 & 0.6127 & 0.2278 & 0.0163 \\
\hline & & Standard error & 0.0632 & 0.0967 & 0.0374 & 0.0025 \\
\hline & & $r^{2}$ value & 0.9975 & 0.9907 & 0.9368 & 0.9906 \\
\hline & \multirow{2}{*}{$\begin{array}{l}\text { Direct Interpolation } \\
\text { (Akima IMSL) }\end{array}$} & Uncertainty & 0.3133 & 0.0714 & 0.1054 & 0.2742 \\
\hline & & Standard error & 0.0221 & 0.0050 & 0.0074 & 0.0193 \\
\hline \multirow{5}{*}{$\begin{array}{l}\text { Proposed } \\
\text { method (2010) }\end{array}$} & \multirow{3}{*}{$\begin{array}{l}\text { Polynomial Curve-fit } \\
\text { (4th order Regression) }\end{array}$} & Uncertainty & 0.3800 & 0.6099 & 0.1758 & 0.0199 \\
\hline & & Standard error & 0.0597 & 0.0962 & 0.0124 & 0.0031 \\
\hline & & $r^{2}$ value & 0.9979 & 0.9910 & 0.9761 & 0.9938 \\
\hline & \multirow{2}{*}{$\begin{array}{l}\text { Direct Interpolation } \\
\text { (Akima IMSL) }\end{array}$} & Uncertainty & 0.2465 & 0.0678 & 0.0051 & 0.0020 \\
\hline & & Standard error & 0.0175 & 0.0048 & 0.0004 & 0.0001 \\
\hline
\end{tabular}

\section{Conclusion}

The paper attempts to investigate a three pressure normalization techniques in combination with three data reduction techniques reported in the multi-hole pressure probe literatures. A particular set of calibration data for a five-hole probe was generated experimentally and used the same data set for analyzing the every possible combination of pressure normalization and data reduction techniques. An additional pressure reduction technique is also proposed in this paper, where the effect of centre-hole pressure is included in all defining pressure normalization parameters. The conclusion may be written as under:

- Out of polynomial curve fit and rational function interpolation methods (which is not discussed in this paper), the former reported better results based on 'goodness of fit' $\left(r^{2}\right)$ values.

- Otherwise, Akima IMSL technique, being a direct interpolation method, passes through all data points, and thereby reported best curve fitting.

- Considering polynomial curve fit method for all the four pressure normalization techniques, the new technique proposed in this paper reported maximum $r^{2}$ values for pitch angle $\left(r^{2}=0.9979\right)$, yaw angle $\left(r^{2}=0.9910\right)$, total pressure $\left(r^{2}=0.9761\right)$ and static pressure $\left(r^{2}=0.9938\right)$. 
A detailed statistical analysis suggests that the proposed pressure normalization technique while combined with fourthorder regression analysis gives the best calibration results. Even the proposed pressure normalization technique performs better than the technique suggested by Gallington (1980) on a same calibration data set.

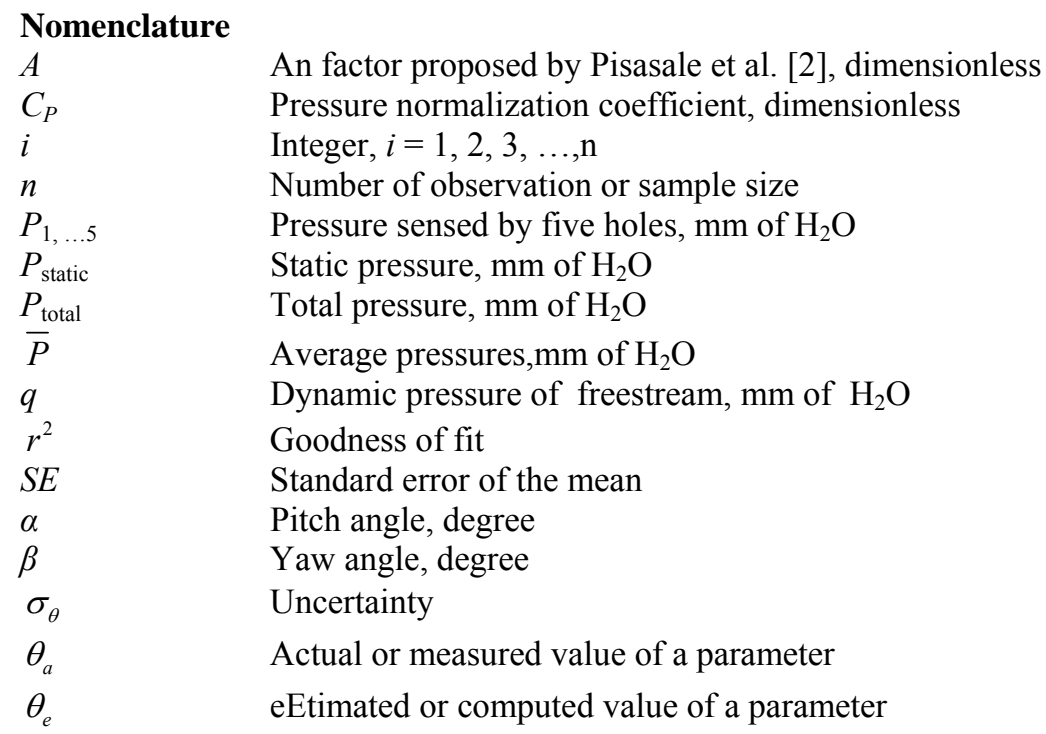

\section{Acknowledgement}

The financial support provided by the Department of Science \& Technology (DST), Govt. of India through its DST-FIST programme is gratefully acknowledged. The authors also like to put on record the infrastructural support rendered by the MNNIT, Allahabad to carry out this project. This paper was presented at the $5^{\text {th }}$ National Conference on Applicable Mathematics in Wave Mechanics and Vibrations (WMVC-2010) held at Kakatiya University, Warangal, India, 13-15 March, 2010.

\section{References}

Bryer, D. W. and Pankhurst, R. C., Pressure-probe methods for determining wind speed and flow direction, Her Majesties Stationery Office, London, 1971.

Gallington, R. W., Measurement of very large flow angles with non-nulling seven-hole prob, Aeronautics Digest, USAFA-TR-8017, 1980, pp. 60-88.

Gerner, A A. and Maurer, C. L., Non-nulling seven-hole probes for high-angle flow measurement, Experiments in Fluids, Vol. 2 , 1984, pp. 95-103.

Krause, L. N. and Dudzindki, T. J., Flow-direction measurement with fixed position probes in subsonic flows over a range of Reynolds number, NASA TMX-52576, 1969.

Pisasale, A. J. and Ahmed, N. A., A novel method for extending the calibration range of five-hole probe for highly threedimensional flows. Flow Measurement and Instrumentation, Vol. 13, 2002, pp.23-30.

Sitaram, N. and Treaster, A. L., A simplified method of using four-hole probes to measure three-dimensional flow fields. J. of Fluids Engineering, Vol. 107, 31, 1985.

Treaster, A. L. and Yocum, A. M., The calibration and application of five-hole probes, Instrumentation Society of America Trans. Vol. 18, 3, 1979, pp. 23-34.

Westphal, R. V., Pauley, W.R. and Eaton, J. K., Interaction between a vortex and a turbulent boundary layer. Part 1: Mean flow evolution and turbulence properties, NASA TM-88361, 1987.

Zillac, G. G., Modeling, calibration, and error analysis of seven-hole pressure probes, Experiments in Fluids, Vol. 14, 1993, pp. $104-120$.

\section{Biographical notes}

Akshoy Ranjan Paul is an Assistant Professor in the Department of Applied Mechanics, Motilal Nehru National Institute of Technology, Allahabad, India. He has received M. E. degree from Jadavpur University, Kolkata, India in 2002. He has more than nine years of experience in teaching and research. His current research area includes internal flow aerodynamics, flow control and turbulence modeling. He has published four textbooks and many research papers in national and international conferences and journals. He also visited a few technical universities in UK, Denmark, and Sweden for academic purposes and technical training. 
Ravi Ranjan Upadhyay is an undergraduate student of Mechanical Engineering discipline at Motilal Nehru National Institute of Technology, Allahabad, India. $\mathrm{He}$ is currently in final year of this study and is doing research in the flow control group of the institute. A few research papers in national and international conferences have been published to his credit.

Dr. Anuj Jain is a Professor in the Department of Applied Mechanics, Motilal Nehru National Institute of Technology, Allahabad, India. He has received the PhD degree IIT Roorkee, India. He has more than 23 years of experience in teaching, research and two years in industry. His current area of research includes thermofluid dynamics in cyclones, internal flow aerodynamics and computational fluid dynamics. He has published more than thirty papers in many interdisciplinary areas. He has delivered a number of invited lectures in several faculty development programmes and conferences. He has visited several universities in USA for academic and research collaborations. He is life member of Indian Society of Technical Education, India, Institution of Engineers (India), Computer Society of India, Indo-US Collaboration of Engineering Education.

Received September 2010

Accepted February 2011

Final acceptance in revised form March 2011 